\title{
A single-use negative-pressure wound therapy device can reduce mastectomy skin flap necrosis in direct-to- implant breast reconstruction
}

\author{
Ji Hun Kim ${ }^{1}$, Yun Sang Kim', \\ Yang Woo Kim ${ }^{1}$, Yu Jin Kim ${ }^{1}$, \\ Yong Soon Chun', \\ Heung Kyu Park², \\ Young Woo Cheon ${ }^{1}$ \\ Departments of ${ }^{1}$ Plastic and \\ Reconstructive Surgery and ${ }^{2}$ General \\ Surgery, Gil Medical Center, Gachon \\ University College of Medicine, Incheon, \\ Korea
}

\begin{abstract}
Background Mastectomy flap necrosis is a common and challenging complication of direct-to-implant (DTI) breast reconstruction. The PICO single-use negative-pressure wound therapy device may reduce the complications associated with skin flap necrosis. We evaluated the relationship between PICO use and the incidence of mild, moderate, and severe skin flap necrosis in patients at high risk of necrosis.

Methods Using medical records from January 2015 to March 2019, we retrospectively analyzed patients who underwent DTI breast reconstruction after oncological breast surgery at a single institution and identified those at high risk for skin flap necrosis. During this period, $\mathrm{PICO}$ was used selectively for patients deemed to be at particularly high risk. Patient demographics, operative characteristics, and the degree of skin flap necrosis were compared according to whether PICO was used.

Results Of 117 patients (122 breasts), 45 were deemed to be at high risk of skin flap necrosis. PICO was applied to 38 of these breasts, and seven breasts received a conventional dressing. Skin flap necrosis occurred in 30 breasts (24 in the PICO group [63.2\%] vs. 6 in the no-PICO group [85.7\%]). Significantly fewer cases of severe skin flap necrosis were observed in the PICO group (2/38 [5.3\%]) than in the no-PICO group (3/7 [42.9\%]) $(P=0.004)$. There were no significant between-group differences in outcomes measured using BREAST-Q scores.

Conclusions Use of a single-use negative-pressure wound therapy device can reduce the risk of severe skin flap necrosis in patients at high risk of skin flap complications associated with DTI breast reconstruction.
\end{abstract}

Keywords Negative-pressure wound therapy / Breast reconstruction / Breast implants / Complications / Necrosis
Received: Aug 19, 2019 Revised: Oct 30, 2019 Accepted: Oct 30, 2019 Correspondence: Young Woo Cheon Department of Plastic and Reconstructive Surgery, Gil Medical Center, Gachon University College of Medicine, 21 Namdong-daero 774beon-gil, Namdong-gu, Incheon 21565, Korea

Tel: +82-32-460-2770, Fax: +82-32-461-2774,

E-mail: youngwooc@gmail.com

Copyright @ 2020 The Korean Society for Aesthetic Plastic Surgery.

This is an Open Access article distributed under the terms of the Creative Commons Attribution Non-Commercial License (https://creativecommons.org/licenses/by-nc/4.0/) which permits unrestricted non-commercial use, distribution, and reproduction in any medium, provided the original work is properly cited. $\quad w w w . e-a a p s . o r g$

\section{INTRODUCTION}

Mastectomy skin flap necrosis is a challenging complication that makes wound management more difficult in patients who undergo breast reconstruction. Overall, the reported incidence of skin flap necrosis ranges from $1.5 \%$ to $41.2 \%$ of breast reconstruction cases [1]. A higher body mass index (BMI), use of immediate breast reconstruction, use of a nipple-sparing mastectomy, a history of smoking, hypertension, diabetes, or exposure to preoperative radiation, or some combination of these factors are correlated with an increased risk of skin flap necrosis [2].

Mastectomy skin flap necrosis requires wound treatment that 
usually includes debridement of the necrotic area. A prospective study found that of 606 consecutive mastectomies, 14\% (85/606) displayed some level of skin flap necrosis. Of these cases, 36\% (31/85) were severe, $7 \%$ (6/85) were moderate, and 54\% (46/85) were mild. Skin flap necrosis was defined as severe if it required operative debridement, resulted in implant loss, or had not healed by 8 weeks after surgery [2]. Complications such as these can increase hospital costs and increase patients' dissatisfaction with the surgical outcome. Skin flap necrosis can also delay postoperative chemotherapy, which may lead to further delays in the patient's overall treatment schedule $[2,3]$. Thus, identifying methods to reduce the risk of skin flap necrosis can help patients obtain appropriate treatment.

Negative-pressure wound therapy (NPWT) is a dressing technique used to treat open wounds by expediting healing by secondary intention [4]. Studies of NPWT in the treatment of closed surgical wounds such as postoperative incisions found decreases in the incidence of surgical-site infections and other surgical-site complications, including dehiscence [5]. In patients who undergo direct-to-implant (DTI) breast reconstruction, the risk of postoperative infection is increased by factors such as smoking, obesity, previous chemotherapy, and prolonged drain use ( $>3$ weeks) [6]. Implant-associated infections can result in prolonged antibiotic use, additional surgical treatment, and implant removal [7]. Therefore, the application of an NPWT device as a preventive or treatment option for mastectomy flap necrosis has been examined [8]. The results of these studies suggest that the use of such a device is an effective treatment strategy.

Studies have examined the effects of NPWT device usage in patients who undergo breast reconstruction [8,9]. However, prior
NPWT devices are large and heavy, and the noise from the large motor interferes with everyday activities and makes it difficult to maintain the device on an outpatient basis [10]. PICO (Smith \& Nephew, Hull, UK), a single-use NPWT device, was developed to address these disadvantages. Compared with traditional NPWT devices, the PICO device is smaller and has advantages typically associated with ambulatory systems [10]. The application of the PICO device for reduction mammoplasty has been examined. However, the use of this device in cases of breast reconstruction that include a mastectomy flap, with its associated risk of skin flap necrosis, has not been well-studied [11].

This retrospective study aimed to investigate the outcomes of use of a PICO device in patients at high risk of skin flap necrosis after DTI breast reconstruction.

\section{METHODS}

This study was a single-site, retrospective review of records for adult female patients who underwent DTI breast reconstruction after skin-sparing mastectomy between January 2015 and March 2019. De-identified patient data were used in accordance with the principles outlined in the Declaration of Helsinki. Patient records were extracted for analysis if they included a 90-day follow-up period within the date range previously specified. Preoperative data regarding demographic and anthropometric variables (age, weight, height, and BMI) and certain clinical and surgical factors (smoking history, axillary clearance, chemotherapy, and hormonal therapy) were collected from patients' medical records. Data regarding implant volume and flap thickness were collected from the intraoper-
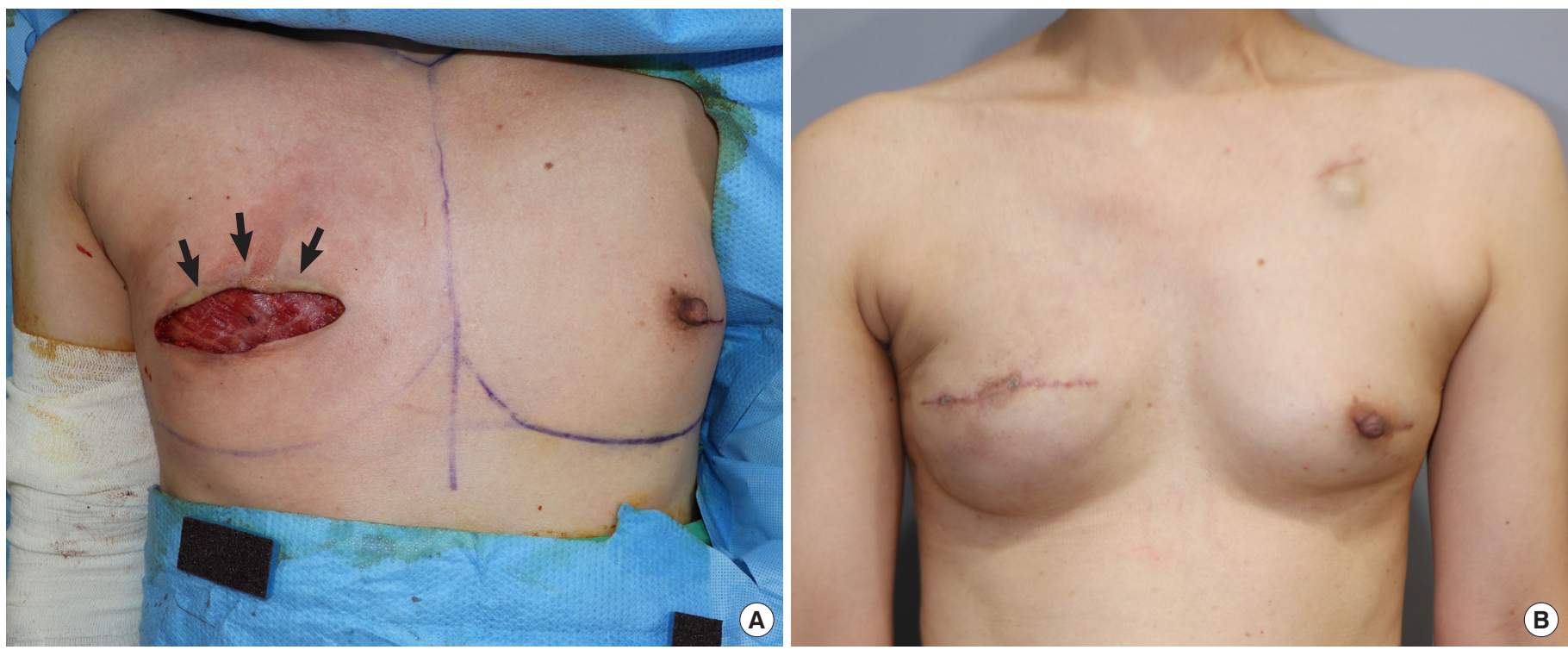

Fig. 1. Visible post-mastectomy skin congestion in the indicated area. (A) Photograph taken immediately after skin-sparing mastectomy. The upper mastectomy skin flap displays visible skin congestion with necrotic changes (black arrows). (B) Representative photograph of long-term follow-up (6 months after the procedure) for the same patient. 
ative evaluation records. A single plastic surgeon (YWC) performed the breast reconstruction procedures using a standardized technique. This included placement of a subpectoral implant (Mentor; Mentor Worldwide LLC, Santa Barbara, CA, USA) with MegaDerm acellular dermal matrix (L\&C BIO, Seongnam, Korea) on the lower pole of the breast, dissection of the lower and medial pectoralis muscles with electrocautery, and placement of closed-suction drains (15-French Jackson-Pratt polyvinyl chloride round drains with trocars) connected to a reservoir (Hemovac [400 mL]; Zimmer Biomet, Warsaw, IN, USA) in the implant pocket between the inframammary fold and the axilla. In accordance with a prospective study of the skin necrosis risk associated with breast re- construction [2], the patients at high risk of skin necrosis were defined as those who fit any of the following criteria: (1) a history of diabetes; (2) a history of smoking; (3) or a BMI of over $25 \mathrm{~kg} / \mathrm{m}^{2}$.

Among the high-risk patients, a PICO was applied on the mastectomy skin flap if one or more of the following indications were present: there was visible congestion of the flap (Fig. 1), the flap was very thin (usually $<6 \mathrm{~mm}$ ), there was congestive bleeding from the skin edges (Fig. 2), or multiple incisions were made by the breast surgeon (e.g., an inframammary fold incision with a periareolar incision) (Fig. 3). The PICO was placed on the closed surgical incision to cover the mastectomy skin flap in a customized fashion for 7 days. It provided continuous pressure of $-80 \mathrm{mmHg}$, and the dress-
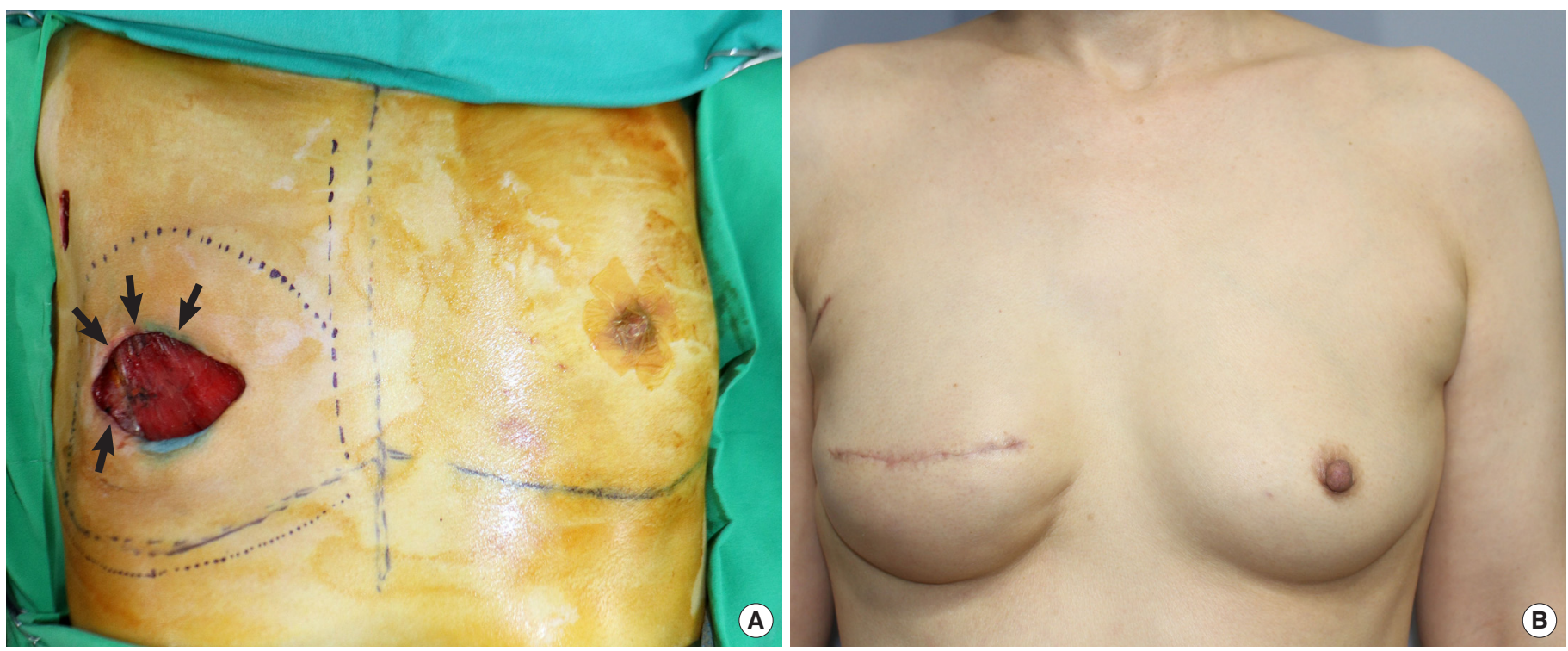

Fig. 2. Congestive bleeding from the mastectomy skin flap edges in the marked area. (A) The upper mastectomy skin flap displays decreased blood circulation and congestive bleeding (black arrows). (B) Representative photograph of long-term follow-up (6 months after the procedure) for the same patient.
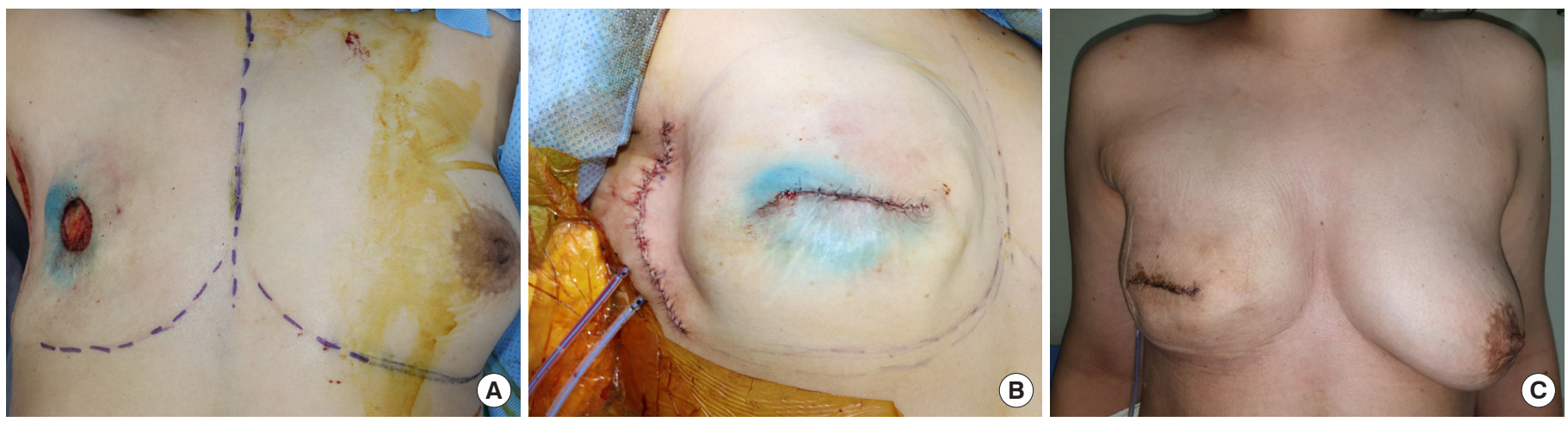

Fig. 3. An example of multiple incisions in skin-sparing mastectomy. (A) A 44-year-old woman underwent skin-sparing mastectomy with two incisions: a lateral incision at the inframammary fold and a periareolar incision. (B) Immediate postoperative photograph of direct-to-implant reconstruction with a silicone gel implant (CPG 321, $340 \mathrm{~mL}$ ) and acellular dermal matrix (Megaderm). (C) Postoperative photograph taken 14 days after the operation. A PICO device was applied for 7 days, and the wound healed without complications. 
ing was changed once every 3 days (Fig. 4) [12]. Beginning 7 days after the operation, a conventional dressing with foam (EasyFoam; CGBio, Seongnam, Korea) was used until the wound was healed. Skin flap necrosis was categorized as mild (fully healed without intervention within 8 weeks after surgery), moderate (managed with outpatient room debridement and fully healed within 8 weeks after surgery), or severe (debridement was performed in the operating room, the implant was lost, or the wound did not fully heal within 8 weeks after surgery) [2].

The drains were removed once the drainage volume was $<40 \mathrm{~mL}$ in 24 hours or on the 14th postoperative day for patients in whom surgery site drainage volume never reached $<40 \mathrm{~mL}$ in 24 hours. This was in agreement with standard guidelines for the timing of drain removal in the field of plastic surgery [6].

The BREAST-Q questionnaire was used to assess the effectiveness and impact of breast surgery from the perspective of the patient [13]. It consists of 42 preoperative and 104 postoperative questions that included domains regarding satisfaction with the breast, physical well-being, psychosocial well-being, and sexual well-being. Preoperative and postoperative BREAST-Q scores were obtained from all patients. The postoperative scores were determined 3 months after the operation, and the differences between the preoperative and postoperative scores were calculated. All statistical analyses were performed using R software, version 3.6.0 (R Foundation for Statistical Computing, Vienna, Austria, 2016). If the results included fewer than 20 patients, the Mann-Whitney test was performed, while the chi-square test was performed if more than 20 patients were included. Two-sided P-values less than 0.05 were considered to indicate statistical significance.
Informed consent was waived, and this investigation was approved by the Institutional Review Board of Gachon University Gil Medical Center (IRB No. GDIRB2019-281).

\section{RESULTS}

During the study period, 122 DTI reconstructions after mastectomy were performed on 117 patients; these reconstructions included 112 unilateral mastectomies and 10 bilateral mastectomies. The median patient age was 48.5 years (range, $28-68$ years), and the median BMI was $23.4 \mathrm{~kg} / \mathrm{m}^{2}$ (range, $18.2-34.9 \mathrm{~kg} / \mathrm{m}^{2}$ ). The demographic characteristics of the patient population are presented in Table 1.

Table 1. Demographic characteristics of all patients

\begin{tabular}{lc}
\hline Characteristic & Total $(\mathrm{n}=122)$ \\
\hline Age $(\mathrm{yr})$ & $48.5(28.0-68.0)$ \\
$\mathrm{BMI}\left(\mathrm{kg} / \mathrm{m}^{2}\right)$ & $23.4(18.2-34.9)$ \\
Smoking history (current or past) & $12(9.8)$ \\
Hypertension & $21(17.2)$ \\
Diabetes & $8(6.6)$ \\
Breast volume (mL) & $295.0(110.0-980.0)$ \\
Neoadjuvant chemotherapy history & $16(13.1)$ \\
Radiotherapy history & $4(3.3)$ \\
Prior breast augmentation & $3(2.5)$
\end{tabular}

Values are presented as median (range) or number (\%). $\mathrm{BMI}$, body mass index.
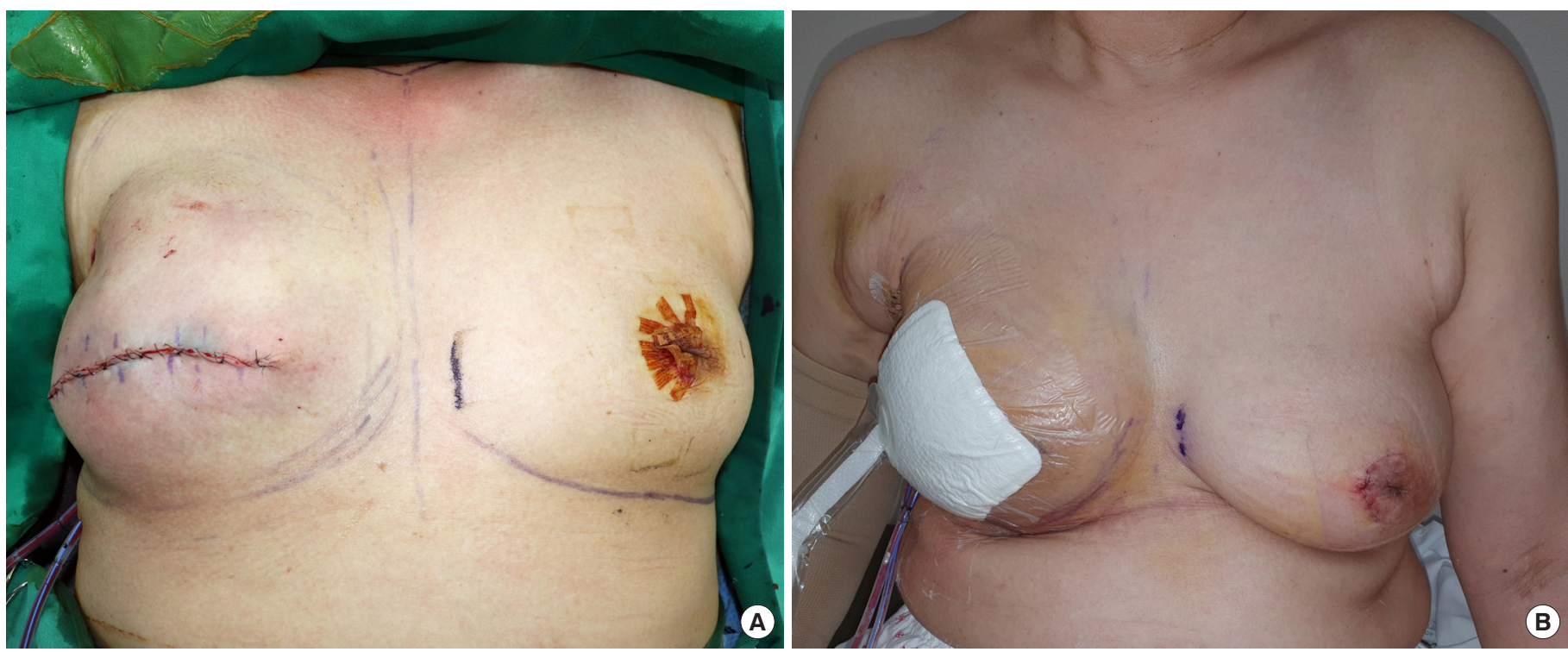

Fig. 4. A PICO device applied to the skin flap of a patient. (A) A 65-year-old woman underwent direct-to-implant breast reconstruction with a silicone gel implant (CPG 321, $355 \mathrm{~mL}$ ) and acellular dermal matrix (Megaderm). (B) A PICO device (15×15 cm) was applied to the surgical incision site, which included a congestive skin-sparing mastectomy skin flap. 
Table 2. Patient demographics and operative characteristics of the PICO and no-PICO groups, both composed of patients at high risk of skin flap necrosis

\begin{tabular}{|c|c|c|c|}
\hline Characteristics & $\operatorname{PICO}(n=38)$ & No-PICO $(n=7)$ & P-value ${ }^{a}$ \\
\hline BMI $\left(\mathrm{kg} / \mathrm{m}^{2}\right)$ & $24.1(19.4-35.1)$ & $23.3(22.2-25.5)$ & $>0.05$ \\
\hline Smoking (current or past) & $5(13.2)$ & $2(28.6)$ & $>0.05$ \\
\hline Hypertension & $7(18.4)$ & $1(14.3)$ & $>0.05$ \\
\hline Diabetes & $5(13.2)$ & $1(14.3)$ & $>0.05$ \\
\hline Specimen weight (g) & $298.3(182.0-1,100.0)$ & $240.5(210.0-910.0)$ & $>0.05$ \\
\hline Implant volume (mL) & $295(155.0-510.0)$ & $260(120.0-475.0)$ & $>0.05$ \\
\hline Neoadjuvant chemotherapy history & $7(18.4)$ & $1(14.3)$ & $>0.05$ \\
\hline Radiotherapy history & $4(10.5)$ & 0 & $>0.05$ \\
\hline Prior breast augmentation & 0 & 0 & $>0.05$ \\
\hline
\end{tabular}

Values are presented as median (range) or number (\%).

$B M I$, body mass index; SLNB, sentinel lymph node biopsy.

alMann-Whitney test.

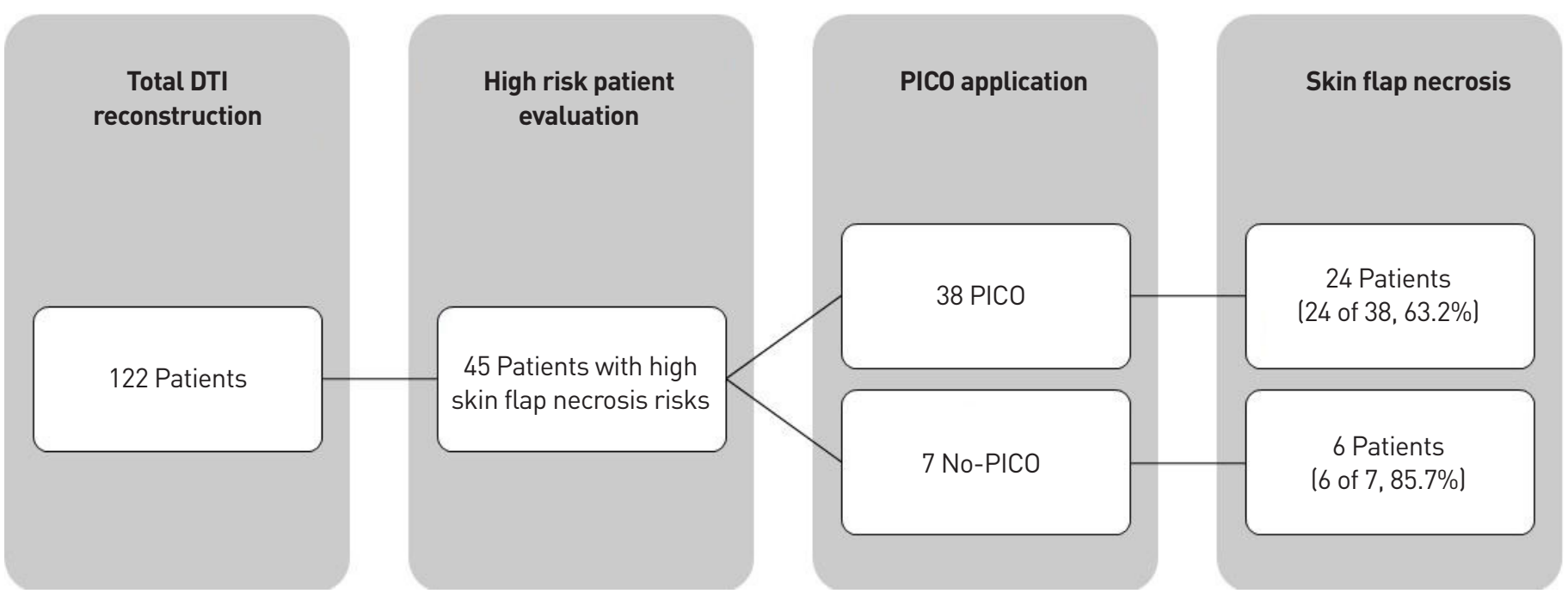

Fig. 5. Flowchart of patients regarding PICO device application status and presence or absence of skin necrosis.

A total of 30 cases of necrosis of the mastectomy skin flap occurred during the study period.

Forty-five patients were found to be at high risk of skin necrosis. Patient demographics and operative characteristics of PICO and no-PICO groups with high skin flap necrosis risk are presented at Table 2. A PICO device was used for 38 mastectomy skin flaps, based on the indications detailed in the Materials and Methods section of this report; the remaining seven skin flaps were treated using conventional dressing. Among the patients who were treated using the PICO device, 24 cases of skin flap necrosis were found, while the other 14 skin flaps in the PICO group healed without complications. Six cases of skin flap necrosis occurred in the patients who did not receive PICO, in whom the flaps were treated using a conventional dressing (Fig. 5). There was no significant difference between the groups with regard to the total skin flap necrosis rate; however, there were differences related to the grade of necrosis.

The results regarding the grade of skin necrosis in patients in the PICO and no-PICO groups are presented in Table 3. A total of 17 skin flaps displayed mild necrosis (15 in the PICO group vs. 2 in the no-PICO group). There were eight cases of moderate skin flap necrosis (7 in the PICO group vs. 1 in the no-PICO group) and 
Table 3. Skin flap necrosis grade and overall necrosis by group

\begin{tabular}{lccc}
\hline Skin flap necrosis & PICO $(n=38)$ & No-PICO $(n=7)$ & P-value $^{\text {a) }}$ \\
\hline Mild & $15(39.5)$ & $2(28.6)$ & $>0.05$ \\
Moderate & $7(18.4)$ & $1(14.3)$ & $>0.05$ \\
Severe & $2(5.3)$ & $3(42.9)$ & $<0.05^{\text {b) }}$ \\
Total & $24(63.2)$ & $6(85.7)$ & $>0.05$ \\
\hline
\end{tabular}

Values are presented as number (\%).

${ }^{\text {al } M a n n-W h i t n e y ~ t e s t ; ~}{ }^{b} \mathrm{P}=0.004$.

five cases of severe skin flap necrosis ( 2 in the PICO group vs. 3 in the no-PICO group). In the PICO group, the skin flap of one patient was debrided in the operating room, and another patient received a smaller implant. In the no-PICO group, the skin flaps of all three patients deemed to have severe necrosis were debrided in the operating room, and no patients experienced implant loss. No statistically significant differences between the groups were found with regard to the rates of mild and moderate skin flap necrosis. However, the rate of severe necrosis was lower in the PICO group than in the no-PICO group, and this result was statistically significant $(\mathrm{P}=0.004)$. There were no statistically significant betweengroup differences in postoperative BREAST-Q scores (315.2 \pm 10.4 in the PICO group vs. $313.8 \pm 17.0$ in the No-PICO group).

\section{DISCUSSION}

Although DTI breast reconstruction is a preferred method of plastic surgeons, management of skin flap necrosis still presents a challenge $[3,14]$. Some studies have evaluated risk factors associated with postoperative complications of breast reconstruction $[6,14$ 17]. Smoking history, a high BMI ( $\left.\geq 25 \mathrm{~kg} / \mathrm{m}^{2}\right)$, immediate breast reconstruction, a nipple-sparing mastectomy, and diabetes have been identified as risk factors for skin flap necrosis $[2,15,16,18]$. When necrosis progresses beyond a certain point, replacement of the implant with a smaller implant or restoration of the area using autologous tissue may be necessary. In our study, we identified patients at high risk of skin flap necrosis, and PICO was selectively applied among these patients based on several indications.

Therefore, as part of ongoing efforts to reduce the rate of skin flap necrosis, the efficacy of PICO application for patients at high risk of skin flap necrosis was examined in this study. The incidence of severe skin flap necrosis was significantly lower in the PICO group than in the no-PICO group (2/38 [5.3\%] vs. 3/7 [43\%], respectively, $\mathrm{P}=0.004)$. Although the total rate of skin flap necrosis was not significantly different between the two groups, the skin flap necrosis rate was lower in the PICO group than in the no-PICO group (63.2\% vs. $85.7 \%)$. Considering that PICO was applied to patients with skin necrosis, this difference may be particularly noteworthy.

NPWT devices have been used to protect against wound infection, dehiscence, and other complications of various types of wounds
$[4,11]$. However, the mechanism of action, especially with regards to tissue perfusion, remains poorly understood. Study findings have indicated that negative-pressure induces an increase in regional blood flow, with beneficial effects including the promotion of angiogenesis, increased perfusion, and reduced edema [19].

The use of closed-incision NPWT has been compared with various other incision types in oncological breast surgery and other types of surgery $[5,20]$. NPWT helps hold the edges of the incision together, removes fluid and infectious materials, and protects the incision from external contamination [20]. These effects provide a reasonable explanation for our results, in which the percentage of cases of severe skin flap necrosis in the PICO group was lower than observed in previous studies (5.3\% vs. $23.8 \%$ [21], 36.5\% [6], and $41.9 \%$ [2]). This result suggests that the use of PICO can reduce the frequency of subsequent operations by decreasing the risk of skin flap necrosis. The economic burden on patients and the psychological stress associated with additional procedures will therefore also diminish.

The use of PICO devices has other advantages that affect their utility for outpatient use. Previous-generation NPWT devices are not easily portable because they are large and heavy. They also have large, noisy motors $[10,22]$. The single-use NPWT system (PICO) was developed to overcome these limitations. It is smaller and more portable than the traditional NPWT devices, which allows for increased mobility and use in an outpatient setting [23]. Like conventional NPWT devices, it can maintain steady sub-atmospheric pressures on the wound within the therapeutic range of -50 to -100 mmHg; patients treated with PICO also experience less pain during dressing changes $[10,24]$. Therefore, based on the four indications given in the Materials and Methods section, we applied the PICO system to DTI mastectomy skin flaps instead of using a conventional NPWT device in appropriate cases. During the study period, there was good patient compliance in the PICO group, and no patient refused to use the device.

The overall rate of skin flap necrosis after mastectomy with reconstruction was $24.6 \%$ (30/122). This rate is higher than that found in other studies, but given the fact that DTI reconstruction is associated with a high skin flap necrosis rate, this result is consistent with previous results $[2,3,8,25]$. The rate of severe skin flap necrosis was $4.1 \%(5 / 122)$ in the entire patient population, which is also consistent with other study results [2].

Additionally, the lack of statistically significant differences in postoperative BREAST-Q scores between the PICO and no-PICO groups could be interpreted as indicating similar patient satisfaction between the two groups.

This study had some limitations. Because a retrospective design was used, we could not evaluate data omitted from the medical records (e.g., ptosis and intraoperative bleeding) that could function as additional risk factors for mastectomy flap necrosis. There was also a relatively large difference in the sample size between the PICO 
group and the no-PICO group (38 patients vs. 7 patients, respectively).

DTI breast reconstruction using an acellular dermal matrix has been increasingly utilized for immediate breast reconstruction. However, mastectomy flap necrosis is an obstacle that remains to be overcome. This study found that the use of PICO in patients who underwent DTI breast reconstruction significantly reduced the incidence of severe skin flap necrosis. This reduction can help lower the rates of reoperation due to skin flap necrosis after DTI breast reconstruction. Future prospective randomized controlled trials with large sample sizes are needed to clarify the effectiveness of PICO for this purpose.

\section{NOTES}

\section{Conflict of interest}

No potential conflict of interest relevant to this article was reported.

\section{Ethical approval}

The study was approved by the Institutional Review Board of Gachon University Gil Medical Center (IRB No. GDIRB2019-281) and performed in accordance with the principles of the Declaration of Helsinki.

\section{Patient consent}

The patients provided written informed consent for the publication and the use of their images.

\section{ORCID}

\section{Ji Hun Kim}

Yun Sang Kim

Yu Jin Kim

Yang Woo Kim

Young Woo Cheon

Yong Soon Chun

Heung Kyu Park https://orcid.org/0000-0002-1947-2476

https://orcid.org/0000-0001-5412-4322

https://orcid.org/0000-0003-1333-4977

https://orcid.org/0000-0002-9823-9567

https://orcid.org/0000-0003-2940-292X

https://orcid.org/0000-0002-7094-677X

https://orcid.org/0000-0003-1897-4228

\section{REFERENCES}

1. Rinker B. A comparison of methods to assess mastectomy flap viability in skin-sparing mastectomy and immediate reconstruction: a prospective cohort study. Plast Reconstr Surg 2016;137:395-401.

2. Matsen CB, Mehrara B, Eaton A, et al. Skin flap necrosis after mastectomy with reconstruction: a prospective study. Ann Surg Oncol 2016; 23:257-64.

3. Robertson SA, Jeevaratnam JA, Agrawal A, et aI. Mastectomy skin flap necrosis: challenges and solutions. Breast Cancer (Dove Med Press) 2017;9:141-52.

4. Argenta LC, Morykwas MJ. Vacuum-assisted closure: a new method for wound control and treatment: clinical experience. Ann Plast Surg
1997;38:563-76.

5. Semsarzadeh NN, Tadisina KK, Maddox J, et al. Closed incision negative-pressure therapy is associated with decreased surgical-site infections: a meta-analysis. Plast Reconstr Surg 2015;136:592-602.

6. Phillips BT, Wang ED, Mirrer J, et al. Current practice among plastic surgeons of antibiotic prophylaxis and closed-suction drains in breast reconstruction: experience, evidence, and implications for postoperative care. Ann Plast Surg 2011;66:460-5.

7. Gabriel A, Sigalove S, Sigalove N, et al. The impact of closed incision negative pressure therapy on postoperative breast reconstruction outcomes. Plast Reconstr Surg Glob Open 2018;6:e1880.

8. Kim DY, Park SJ, Bang SI, et al. Does the use of incisional negativepressure wound therapy prevent mastectomy flap necrosis in immediate expander-based breast reconstruction? Plast Reconstr Surg 2016; 138:558-66.

9. Spruce P, Leak K, Johnson S. Improving outcomes for patients following surgery for breast cancer. Wounds UK 2019;5:47-55.

10. Payne C, Edwards D. Application of the single use negative pressure wound therapy device (PICO) on a heterogeneous group of surgical and traumatic wounds. Eplasty 2014;14:e20.

11. Galiano RD, Hudson D, Shin J, et al. Incisional negative pressure wound therapy for prevention of wound healing complications following reduction mammaplasty. Plast Reconstr Surg Glob Open 2018;6:e1560.

12. Malmsjo M, Huddleston E, Martin R. Biological effects of a disposable, canisterless negative pressure wound therapy system. Eplasty 2014;14: e15.

13. Pusic AL, Klassen AF, Scott AM, et al. Development of a new patientreported outcome measure for breast surgery: the BREAST-Q. Plast Reconstr Surg 2009;124:345-53.

14. Sue GR, Long C, Lee GK. Management of mastectomy skin necrosis in implant based breast reconstruction. Ann Plast Surg 2017;78(5 Suppl 4):S208-11.

15. Colwell AS, Damjanovic B, Zahedi B, et al. Retrospective review of 331 consecutive immediate single-stage implant reconstructions with acellular dermal matrix: indications, complications, trends, and costs. Plast Reconstr Surg 2011;128:1170-8.

16. Hultman CS, Daiza S. Skin-sparing mastectomy flap complications after breast reconstruction: review of incidence, management, and outcome. Ann Plast Surg 2003;50:249-55.

17. Chen CF, Lin SF, Hung CF, et al. Risk of infection is associated more with drain duration than daily drainage volume in prosthesis-based breast reconstruction: a cohort study. Medicine (Baltimore) 2016;95: e5605.

18. McCarthy CM, Disa JJ, Pusic AL, et al. The effect of closed-suction drains on the incidence of local wound complications following tissue expander/implant reconstruction: a cohort study. Plast Reconstr Surg 2007;119:2018-22.

19. Suh H, Lee AY, Park EJ, et al. Negative pressure wound therapy on closed surgical wounds with dead space: animal study using a swine model. Ann Plast Surg 2016;76:717-22. 
20. Ferrando PM, Ala A, Bussone R, et al. Closed incision negative pressure therapy in oncological breast surgery: comparison with standard care dressings. Plast Reconstr Surg Glob Open 2018;6:e1732.

21. Sue GR, Lee GK. Mastectomy skin necrosis after breast reconstruction: a comparative analysis between autologous reconstruction and implant-based reconstruction. Ann Plast Surg 2018;80(5S Suppl 5): S285-7.

22. Sposato G, Molea G, Di Caprio G, et al. Ambulant vacuum-assisted closure of skin-graft dressing in the lower limbs using a portable miniVAC device. Br J Plast Surg 2001;54:235-7.
23. Nherera LM, Trueman P, Karlakki SL. Cost-effectiveness analysis of single-use negative pressure wound therapy dressings (sNPWT) to reduce surgical site complications (SSC) in routine primary hip and knee replacements. Wound Repair Regen 2017;25:474-82.

24. Birke-Sorensen H, Malmsjo M, Rome P, et al. Evidence-based recommendations for negative pressure wound therapy: treatment variables (pressure levels, wound filler and contact layer): steps towards an international consensus. J Plast Reconstr Aesthet Surg 2011;64 Suppl:S1-16.

25. Huang Y, Jogeland K, Samuelsson M, et al. Management of severe skin flap necrosis after mastectomy: case report. J Surg 2019;2019:1184. 